\title{
Recovering thermodynamic consistency of the antitrapping model: A variational phase-field formulation for alloy solidification
}

\author{
Angbo Fang ${ }^{1, *}$ and Yongli $\mathrm{Mi}^{1,2, \dagger}$ \\ ${ }^{1}$ Department of Chemistry, Tongji University, Shanghai, P. R. China 200092 \\ ${ }^{2}$ Department of Chemical and Biomolecular Engineering, Hong Kong University of Science and Technology, Kowloon, Hong Kong
}

(Received 13 May 2012; revised manuscript received 25 October 2012; published 3 January 2013)

\begin{abstract}
The phenomenological antitrapping phase-field model has attained much success in describing alloy solidification. The heuristically introduced antitrapping current enables removing artificial effects due to the use of large interfacial width. Nevertheless, such a model is not thermodynamically consistent and has not been fitted into a variational framework. Here we present two approaches to develop a variational phase-field model to describe patten formation in alloys. Following the principles of linear irreversible thermodynamics we build in the cross-coupling between the phase transition rate and solute diffusion current. Our formulation not only naturally incorporates the antitrapping current but also predicts the conjugated mesoscopic solute drag effect. A more general form of the antitrapping current is obtained by thin-interface analysis. Benchmark simulations on isothermal dendrite growth are carried out to show the capability of our model to quantitatively characterize the interface evolution and solute profile even with a large interface width used. Importantly, our theory also provides general insights on how to obtain the genuine dynamic coupling between nonconserved and conserved order parameters. This leads to a thermodynamically consistent generalization of the celebrated model C proposed by Hohenberg and Halperin [Rev. Mod. Phys. 49, 435 (1977)].
\end{abstract}

DOI: 10.1103/PhysRevE.87.012402

PACS number(s): 81.10.Aj, 64.70.dg, 89.75.Kd

\section{INTRODUCTION}

The phase-field approach has been increasingly used as an efficient means to study problems involving evolving interfaces, with great success achieved in modeling solidification phenomena [1-4]. It is advantageous over the traditional sharp-interface method by avoiding explicitly tracking phase boundaries. In this approach the macroscopic zero-thickness (sharp) interface is resolved mesoscopically as a region with finite thickness $\sim W$, which should be much smaller than the characteristic length scale in bulk phases. However, in realistic simulations it can be chosen much larger than the physical interface thickness as long as artificial effects caused by this scale-up can be controlled.

The "thin-interface" (TI) analysis [5] has been invented to achieve this control for the solidification problem. It has proven a great success in quantitatively modeling of dendritic growth in pure melts at low undercooling. Nevertheless, for the more realistic situation with asymmetric transport properties in the liquid and solid, this scheme is insufficient to remove all the artificial scale-up effects [6]. For a dilute binary alloy with negligible diffusivity in the solid, Karma [7] introduced a phenomenological antitrapping (AT) current to the solute diffusion equation and attained this control within TI limit. Ohno and Matsuura [8] further devised it to accommodate finite diffusivity in the solid. Recently it has also been adapted to multicomponent systems [9].

The phenomenologial AT models cannot be derived from a Lyapounov functional, and thermodynamic consistency is not satisfied at the mesoscopic level. In this work, by employing

\footnotetext{
*afang1@gmail.com

†Corresponding author: keymix@ust.hk
}

Onsager's variational approach to linear irreversible thermodynamics (LIT), we develop a new phase-field model with full thermodynamic consistency. In our model the solute transport equation has a kinetic current, and the phase-field equation gains a new contribution describing the mesoscopic solute drag effect. Both "solute (anti-)trapping" and "solute drag" arise from the dynamic cross-coupling between phase transition and solute diffusion. Simulations on two-dimensional isothermal dendrite growth show that our model can quantitatively and efficiently describe the solidification process, with reasonably large interface width used.

\section{FORMULATION}

\section{A. The traditional phase field model and antitrapping current}

For clarity and simplicity, we focus on the problem of isothermal solidification [10] for a dilute binary alloy of A and $\mathrm{B}$. We first introduce relevant notations and show how the standard variational phase-field model can be obtained via Onsager's approach. The system is described by the two scalar fields: $\phi$ is the scalar phase field introduced to distinguish between solid $(\phi=+1)$ and liquid $(\phi=-1)$ phase of the major component $\mathrm{A}$ and $c$ is the concentration of the minor component B (solute). The free energy functional of the system is

$$
F=\int_{d V}\left[\frac{1}{2} H W^{2}|\nabla \phi|^{2}+f_{0}(\phi)+f_{A B}(\phi, c, T)\right],
$$

where $f_{0}(\phi)=H\left(-\phi^{2} / 2+\phi^{4} / 4\right)$ is the double-well potential with a barrier height $H$ and $f_{A B}=g(\phi) f_{s}\left(c_{s}\right)+[1-$ $g(\phi)] f_{l}\left(c_{l}\right)$ is a functional that interpolates between the free-energy density of solid and liquid bulk phases, with $g(\phi)=15\left(\phi-2 \phi^{3} / 3+\phi^{5} / 5\right) / 8$ the standard choice. Here $c_{s}$ and $c_{l}$ are, respectively, the concentration field in the solid and liquid and are related via the condition of equal chemical 
potential [11]: $\partial f_{s} / \partial c_{s}=\partial f_{l} / \partial c_{l}$. The concentration field $c$ is determined by the mixture rule as $c=\frac{1+h(\phi)}{2} c_{s}+\frac{1-h(\phi)}{2} c_{l}$, with $h(\phi)$ another interpolating function satisfying $h( \pm 1)=$ \pm 1 . In variational formulation, $h(\phi)$ should be identical to $g(\phi)$. The rate of energy dissipation can be expressed as $-\dot{F}=-\int_{d V}[(\delta F / \delta \phi) \dot{\phi}+(\delta F / \delta c) \dot{c}]$, where a dot on a variable denotes its time-varying rate of change. For our system characterized by the nonconserved field $\phi$ and the conserved field $c$, it is natural to choose $\dot{\phi}$ and $\mathbf{J}$ as the appropriate fluxes, with $\mathbf{J}$ defined by the mass conservation law $\dot{c}+\nabla \cdot \mathbf{J}=0$. As restricted by Curie's principle, no coupling is allowed between $\dot{\phi}$ and $\mathbf{J}$. Hence the dissipation potential can be constructed as $\Psi_{0}=\dot{\phi}^{2} / 2 K_{\phi}+\mathbf{J}^{2} / 2 M_{c}$, with $K_{\phi}$ and $M_{c}$ the mobility coefficients describing the relaxation or transport of $\phi$ and $c$, respectively. Following Onsager, we can derive the constitutive equations by setting the variation of $-\dot{F}-\Psi_{0}$ with respect to the variation of fluxes to zero. By this procedure the equations of motion for the phase field and solute concentration are obtained as

$$
\dot{\phi}=-K_{\phi} \frac{\delta F}{\delta \phi}
$$

and

$$
\dot{c}=\nabla \cdot\left(M_{c} \nabla \mu\right),
$$

where the second equation is obtained by plugging into the mass conservation law the constitutive relation $\mathbf{J}=$ $-M_{c} \nabla \mu \equiv \mathbf{J}_{c}$, with $\mu \equiv \delta F / \delta c$ is the chemical potential. This set of equations is the regular variational phase-field formulation.

Equations (2)-(3) are thermodynamically consistent but less useful than the AT model due to the magnified artificial effects when a computationally trackable $W$ is used. To counteract the artificial solute trapping in realistic simulations, Karma [7] proposed the AT current

$$
\mathbf{J}_{a t}=-a(\phi) W\left(c_{l}^{0}-c_{s}^{0}\right) \dot{\phi} \frac{\nabla \phi}{|\nabla \phi|}
$$

to amend the diffusion flux $\mathbf{J}_{c}$, where $c_{l}^{0}\left(c_{s}^{0}\right)$ are the equilibrium concentrations in the liquid (solid) at a fixed temperature and $a(\phi)$ is an interpolating function to be constrained by TI analysis. The form of $\mathbf{J}_{a t}$ is motivated by the observation that to lowest order, the solute trapping effect is proportional to the interface migration speed $\mathbf{V}$ and in the TI limit $\mathbf{V} \sim-W \dot{\phi} \nabla \phi /|\nabla \phi|$. The analysis of Eq. (4) leads us to consider if there can be genuine dynamic coupling between $\phi$ and $c$. If so, we may obtain a thermodynamically consistent phase-field model with the AT current naturally emerging from the general principle of LIT other than heuristically introduced. We mention that the importance of the dynamic cross-coupling and Onsager symmetry in phase-field models of solidification was also suggested in a recent paper by Brener and Temkin [12].

In the following two subsections we present two approaches to obtain the new variational phase-field model: The first is derived from a more formal point of view, while the second is mainly based on nonequilibrium thermodynamic analysis of the interfacial solute transport.

\section{B. The first approach to our model}

In the first approach, we note that while $\dot{\phi}$ is sufficient to locally describe the rate of liquid-solid transformation, as a scalar it does not specify the growth direction at the mesoscopic scale. We may amend a director $\mathbf{n}_{\phi} \equiv \nabla \phi /|\nabla \phi|$ to characterize the local growth direction. Because $\mathbf{n}_{\phi} \cdot \mathbf{n}_{\phi} \equiv 1$, the energy dissipation can be rewritten as $-\dot{F}=-\int_{d V}\left[\left(\delta F / \delta \phi \mathbf{n}_{\phi}\right)\right.$. $\left.\left(\dot{\phi} \mathbf{n}_{\phi}\right)-\mu \nabla \cdot \mathbf{J}\right]$. Hence we can formally identify $\tilde{\boldsymbol{\phi}} \equiv \dot{\phi} \mathbf{n}_{\phi}$ rather than $\dot{\phi}$ as the appropriate flux describing the local phase transition. This procedure of "transforming scalars into vectors" is also strongly motivated by the interfacial forceflux analysis of the corresponding free-boundary problem, as recently discussed by Brener and Temkin [12]. Interestingly, in the model proposed by Wang et al. [13], the entropy density has an explicit dependence on $\nabla \phi$ and the entropy flux contains a contribution proportional to $W \dot{\phi} \nabla \phi$. In the sharp-interface limit this is identified as the entropy flux caused by interface migration. Therefore, in the phase-field modeling where surface excess quantities are smeared as mesoscopic bulk quantities, this entropy flux excess is manifested as a part of entropy production. This, to a certain degree, also justifies $\tilde{\boldsymbol{\phi}}$ as a more appropriate dynamic flux when the interface is moving. The corresponding dissipation potential can be constructed as

$$
\Psi_{1}=\frac{\tilde{\boldsymbol{\phi}}^{2}}{2 K_{\phi}}+\frac{\mathbf{J}^{2}}{2 M_{c}}+\gamma \tilde{\boldsymbol{\phi}} \cdot \mathbf{J},
$$

where $\gamma$ is the cross-coupling coefficient. In general $\gamma$ can depend on $\phi$ and $c$ and should satisfy $\gamma^{2} \leqslant 1 /\left(K_{\phi} M_{c}\right)$ to make $\Psi_{1}$ positive definite. Following Onsager's variational approach and using the continuity equation we obtain the generalized equations of motion:

$$
\begin{gathered}
\left(1-K_{\phi} \tilde{\gamma}^{2} / M_{c}\right) \dot{\phi}=-K_{\phi}\left[\frac{\delta F}{\delta \phi}-\tilde{\gamma} \boldsymbol{n}_{\phi} \cdot \nabla \mu\right], \\
\dot{c}=\nabla \cdot\left[M_{c} \nabla \mu+\tilde{\gamma} \dot{\phi} \boldsymbol{n}_{\phi}\right],
\end{gathered}
$$

where $\tilde{\gamma} \equiv M_{c} \gamma$. Clearly, there are new terms appearing in both equations due to the dynamic coupling between the phase transition and solute diffusion processes. This formulation is by construction thermodynamically consistent.

\section{The second approach to our model}

The second approach starts by performing a macroscopic thermodynamic analysis of the steady growth state. This is nontrivial due to the interplay of solute diffusion and interface attachment kinetics. We find it insightful to plot a circuit diagram for the solute transport, following the entropy flow diagram used in studying the crystallizing process of helium $[14,15]$. From Fig. 1(a) it is clear that the total solute current across the interface $\mathbf{J}_{I}$ is the sum of the dissipative current $\mathbf{J}_{\mathbf{D}}$ through the Kapitza resistance $R_{K}$ and the kinetic current $\tilde{\beta} \mathbf{V}$ convected by the solidifying liquid. The effective growth rate in general depends on the way $\mathbf{J}_{I}$ partitions [12]. For alloy solidification at low undercooling, $\delta \mu=0$. The crucial point is that the Kapitza resistance is negligibly small for the usual crystal-melt interface so that $\mathbf{J}_{D}$ can be freely 


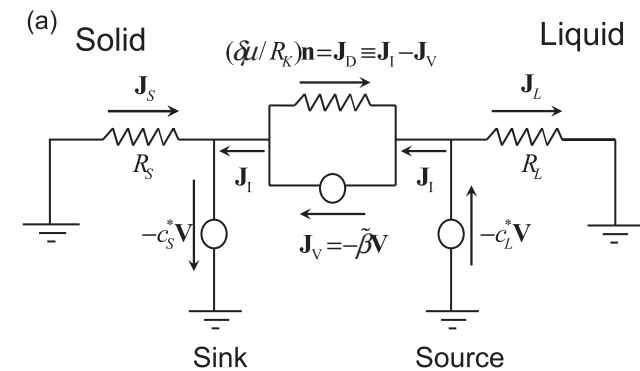

(b)

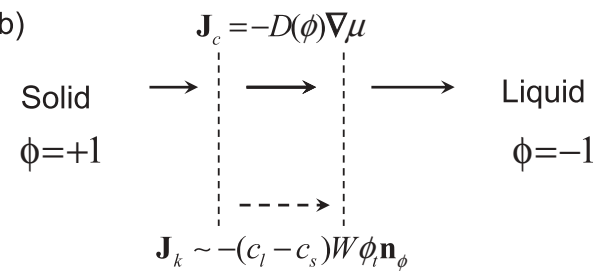

FIG. 1. (a) Diagram of the macroscopic solute flow at the liquidsolid interface with migration speed $\mathbf{V}=V \mathbf{n}$, with $\mathbf{n}$ the interface normal pointing from solid into liquid. The solute concentration of the newly formed solid is supplied by the diffusion flux $\mathbf{J}_{\mathbf{S}}$ from the solid plus the flux $\mathbf{J}_{\mathbf{I}}$ arriving through the interface. Similarly, the solute concentration of the liquid being frozen is removed by $\mathbf{J}_{\mathbf{L}}$ and $\mathbf{J}_{\mathbf{I}}$. (b) Solute flow in the mesoscopic interfacial region of our phase-field model. The dissipative current $\mathbf{J}_{c}$ varies continuously across the interfacial region. A kinetic current, $\mathbf{J}_{k}$, is introduced to rectify the oversinked solute due to the finite interface width. Its form is suggested the mesoscopic representation of $\mathbf{V}$.

adjusted to yield the desired value of $\mathbf{J}_{I}$ and realize the prescribed solute partition according to equilibrium phase diagrams. This is not the case when we go to the mesoscopic level described by phase-field models. At this level, the dissipative solute fluxes, either in the bulk $\left(\mathbf{J}_{S}\right.$ and $\left.\mathbf{J}_{L}\right)$ or at the interface $\left(\mathbf{J}_{D}\right)$, are unified into a single diffusion flux, with the diffusion coefficient $D(\phi)$ varying across the interfacial region. The interface motion is implicitly characterized by the propagation of the $\phi=0$ contour, and the local solute partition is instantly accomplished according to the prescribed mixing rule. However, with the interfacial width becoming finite and the interface motion becoming fast, more solute than desired is left behind the solid-liquid front due to the inhomogeneity of $\dot{\phi}$ across the interfacial region. Going back to the macroscopic level, $\mathbf{J}_{D}$ is no longer at our full disposal. This is because the mesoscopic description specifies the system more strictly than the macroscopic free-boundary problem. TI asymptotic analysis demonstrates that artificial kinetic effects cannot be fully removed by cleverly designing the forms of $g(\phi)$ and $D(\phi)$. Therefore we need to put more freedom into the phase-field model to faithfully reproduce the desired solute partition during interface migration. This can be achieved by explicitly introducing a kinetic rectification $\mathbf{J}_{k}$ to the interfacial solute flux. For this purpose we construct $\mathbf{v}=-\tilde{a}(\phi) W \dot{\phi} \mathbf{n}_{\phi}$ as the local velocity, with $\tilde{a}$ a positive regulating factor. Setting $\mathbf{J}_{k}=\mathbf{v} c$ as the kinetic flux, we have the full mass conservation equation given by

$$
\dot{c}=-\nabla \cdot\left[J_{c}+J_{k}\right] .
$$

It is noted that the form of $\mathbf{J}_{k}$ is similar to the phenomenological AT current. By incorporating $\mathbf{J}_{k}$ the mesoscopic continuity of $c$ is restored at the time scale of phase transition, which becomes smaller than the interfacial diffusion time when $W$ is large. Substantially, this kinetic current can persist even for the case with symmetric transport coefficients in both liquid and solid.

Using Eq. (8) we can recast $\dot{F}$ into the following form:

$$
\dot{F}=\int_{d V}\left[\frac{\delta F}{\delta \phi} \dot{\phi}+\mathbf{J}_{c} \cdot \nabla \mu-\tilde{a} W c\left(\mathbf{n}_{\phi} \cdot \nabla \mu\right) \dot{\phi}\right],
$$

where the last term is not included in traditional phasefield models. The kinetic rectification to solute transport and energy dissipation arises from the dual role played by $\dot{\phi}$ for a moving interface: On the one hand $\dot{\phi}$ itself is a thermodynamic flux, whereas on the other hand $\dot{\phi}$ multiplied by $\mathbf{n}_{\phi}$ is a mesoscopic kinetic variable. In Eq. (9) $\dot{\phi}$ and $\mathbf{J}_{c}$ can be identified as two uncoupled fluxes and $\Psi_{0}$ is the appropriate dissipation potential. We obtain by variational calculus, in addition to $\mathbf{J}_{c}=-M_{c} \nabla \mu$, the following constitutive equation:

$$
\dot{\phi}=-K_{\phi}\left[\frac{\delta F}{\delta \phi}-\tilde{a} W c\left(\mathbf{n}_{\phi} \cdot \nabla \mu\right)\right],
$$

where the second term in the square bracket is readily identified as the mesoscopic solute drag, absent in all previous phase-field models for solidification. Importantly, it should be distinguished from the macroscopic solute drag in rapid solidification processes when solute trapping happens. The direction of this mesoscopic solute drag varies across the interfacial region and thus leads to modulation of the phase transition rate.

Remarkably, the equations of motion [Eqs. (8) and (10)] obtained with this approach can be exactly mapped to Eqs. (6)(7) obtained via the first approach by identifying $\tilde{\gamma}=\tilde{a} W c$ and redefining $K_{\phi}$. This is not surprising because both approaches have effectively taken into account the dynamic cross-coupling between the phase transition and solute diffusion process in the framework of LIT. The first approach is more formal and in some sense more general (e.g., naturally allowing an anisotropic mobility for the phase-field relaxation). However, the second one is physically more transparent, and we adhere to it in the following discussion.

\section{Thin-interface analysis}

Our variational model differs from the phenomenological AT model only by the additional "solute drag" term. This term is crucial because it avoids "action without anti-action" and restores the thermodynamic consistency lacking in AT models. It is important to note that this term does not influence the equilibrium profiles for $\phi$ and $c$. Thus, similar to the KKS model [11] and the AT model [16], the salient feature is maintained that the equilibrium phase-field profile and surface tension are independent of the solute concentration. In fact, the surface tension is given by $\gamma_{s}=\frac{16}{15} a_{1} W H$ with $a_{1} \equiv 5 \sqrt{2} / 8$. To determine $a(\phi)$ and see how solute drag may influence the scale-up effects in realistic calculations, a TI analysis is helpful along the same line as in Refs. [7] and [8]. For this purpose we introduce the dimensionless local supersaturation defined by 
$u=\left(c_{l}-c_{l}^{0}\right) /\left(c_{l}^{0}-c_{s}^{0}\right)$ and measure length in units of $d_{0}$ and time in units of $d_{0}^{2} / D$, with $d_{0}$ the chemical capillary length. The TI analysis uses $\epsilon=W / d_{0}$ as the expansion parameter, and the dimensionless equations are given by

$$
\begin{aligned}
\alpha_{0} \epsilon^{2} \partial_{t} \phi= & \epsilon^{2} \nabla^{2} \phi+\phi-\phi^{3}-\lambda g^{\prime}(\phi) u \\
& +\frac{16}{15} a_{1} \epsilon^{2} a(\phi) \frac{\nabla \phi}{|\nabla \phi|} \cdot \nabla u
\end{aligned}
$$

and

$$
\begin{aligned}
& \frac{[1+k-(1-k) h(\phi)]}{2} \partial_{t} u \\
& =\nabla \cdot\left\{q(\phi) \nabla u+a(\phi) \epsilon[1+(1-k) u] \partial_{t} \phi \frac{\nabla \phi}{|\nabla \phi|}\right\} \\
& \quad+\frac{1}{2}[1+(1-k) u] \partial_{t} h(\phi)
\end{aligned}
$$

with $\alpha_{0}=D /\left(K_{\phi} H W^{2}\right)$ a dimensionless constant, $\lambda=a_{1} \epsilon$, and $2 a(\phi)=\left[\frac{1+k}{1-k}-h(\phi)\right] \tilde{a}(\phi)$. It is immediately seen that the solute drag term is of the order of $\epsilon^{2}$. Nevertheless, further analysis shows that it is actually of the order of $\epsilon^{3}$ because to leading order $u$ is independent of the normal coordinate across the interface. Therefore, the solute drag term does not alter the previous asymptotic results performed to second order of $\epsilon$. With the interpolating functions chosen as $h(\phi)=g(\phi)$ and $q(\phi)=(1-\phi) / 2$, the interface stretching and surface diffusion can be eliminated in the TI limit. To eliminate the chemical potential jump, we have $F^{+}=F^{-}$, with

$$
F^{ \pm}=\int_{0}^{ \pm \infty} d \eta\left[p\left(\phi_{0}\right)-p(\mp 1)\right]
$$

and

$$
p(\phi)=\left[h(\phi)-1-2 a(\phi) \partial_{\eta} \phi\right] / q(\phi),
$$

where $\eta$ is a coordinate normal to the interface scaled by $W$. To obtain a simple expression for $a(\phi)$, Karma and his collaborators proposed the ansatz $p(\phi)=h(\phi)-1$, which yields $a(\phi)=[1-h(\phi)][1-q(\phi)] / \sqrt{2}\left(1-\phi^{2}\right)$. However, we find a more general ansatz: $p(\phi, \alpha)=\alpha(h(\phi)-1)+$ $2(\alpha-1) q(\phi)$, where $\alpha$ is a constant parameter. Note that with the special choice of $1-h(\phi)=2 q(\phi)$ in the nonvariational formulation, our ansatz collapses to the old one for arbitrary $\alpha$. Nevertheless, when $h(\phi)$ and $q(\phi)$ are linearly independent, we can explore the benefit due to the freedom of choice on $\alpha$, without violating any constraints obtained in TI analysis. For $h(\phi)=g(\phi)$ and $q(\phi)=(1-\phi) / 2$, the choice of $\alpha=2$ yields

$$
a(\phi)=\frac{1}{2 \sqrt{2}}\left[1+\frac{1}{4}\left(3 \phi^{2}-7\right) \phi^{2}\right],
$$

which is symmetric with respect to $\phi=0$ and has better numerical performance than other choices of $\alpha$. Importantly, this unique choice renders the kinetic current an odd parity and restores the liquid-solid exchange symmetry of the original solute transport equation. The asymptotic matching with the velocity-dependent Gibbs-Thomson condition gives the following expression for the kinetic coefficient:

$$
\beta=a_{1}\left[\tau /(W \lambda)-a_{2}(\alpha) W / D\right]
$$

where $\tau=1 /\left(K_{\phi} H\right)$ is the phase relaxation time, $a_{2}(\alpha)=$ $\alpha a_{2}(1)+(1-\alpha) a_{2}(0)$, with $a_{2}(1)=0.3981$ and $a_{2}(0)=$ 0.6267 . For the symmetric choice of $a(\phi)$ given by Eq. (15) we have $a_{2}(2)=0.4258 a_{2}(1)$.

It is important to remember that Eq. (16) is not an exact result but an approximation presumably good for small ratio of $W / d$. It was pointed by the work of Brener and Temkin [12] that such a thin-interface limit result should be taken with care. With certain phase-field parameters, it can give rise to a negative kinetic coefficient and lead to unphysical instabilities in the corresponding sharp-interface formulation.

\section{RESULTS AND DISCUSSIONS}

The performance of our model was examined by carrying out two-dimensional simulations of isothermal dendritic growth. Crystalline anisotropy was included by generalizing Eq. (10) to a standard anisotropic form $[5,16]$. The kinetic coefficient becomes $\beta(\theta)=a_{1}\{\tau(\theta) /[W(\theta) \lambda]-$ $\left.a_{2}(\alpha) W(\theta) / D\right\}$, with $W(\theta)=W a_{s}(\theta), a_{s}(\theta)=1+\epsilon_{4} \cos 4 \theta$ the fourfold anisotropy factor, and $\theta \equiv \arctan \left(\partial_{y} \phi / \partial_{x} \phi\right)$. To make $\beta(\theta)$ vanish we require the anisotropic phase relaxation time to satisfy $\tau(\theta)=\tau_{0}(\alpha) a_{s}(\theta)^{2}$ and $\tau_{0}(\alpha)=a_{2}(\alpha) \lambda W^{2} / D$.

We compare the results of our current model to those obtained by the phenomenological AT model. Both models have the desired thin-interface limit, but only our model is thermodynamically consistent and completely captures the dynamic coupling of phase transition and solute diffusion at the mesoscopic level. We use a simple finite-difference method with $\Delta x=0.36$ and $\Delta t=0.008, W=\tau_{0}(1)=1, \epsilon_{4}=0.02$, $k=0.15$. In all simulations, the initial condition consists of a circular solid seed of radius $r=3 \sim 5$ and $u=-0.55$ throughout the simulating region. We further note that, while the second-order TI analysis uniquely determines a phase relaxation time that simply scales $\sim W^{3}$, this is not necessarily good for large $W$ when high-order correction becomes important. Therefore we let $\tau_{0}=\chi \tau_{0}(1)$ and treat $\chi$ as an adjustable parameter [17]. To see how the results can depend on the choice of $\tau_{0}$, we plot in Fig. 2 the dimensionless dendrite tip velocity $V d_{0} / D$ and tip radius $\rho / d_{0}$ of the steady growth state as a function of $\chi$, for $W=3 d_{0}$ and $W=6 d_{0}$. Results are plotted only in a selected section of $\chi$ within which no solute trapping happens, and the tip velocity does not deviate too
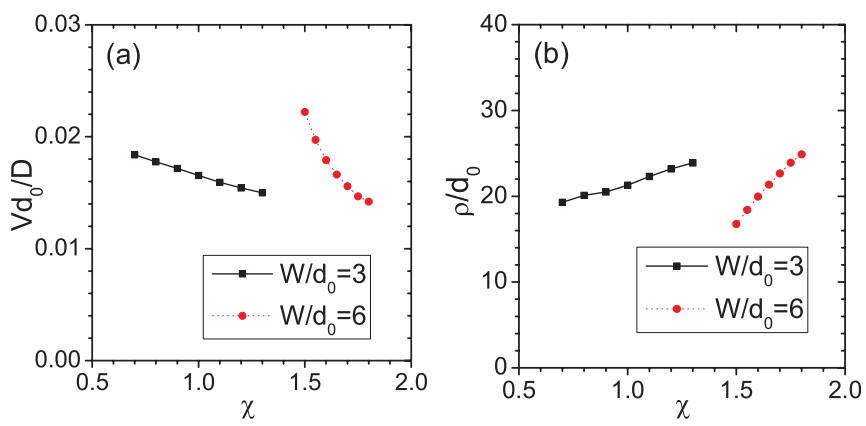

FIG. 2. (Color online) The dimensionless (a) tip velocity $V d_{0} / D$ and (b) tip radius $\rho / d_{0}$ of the steady growth state as a function of the dimensionless phase relaxation time $\chi$ for two interface width $W=3 d_{0}$ and $W=6 d_{0}$. 

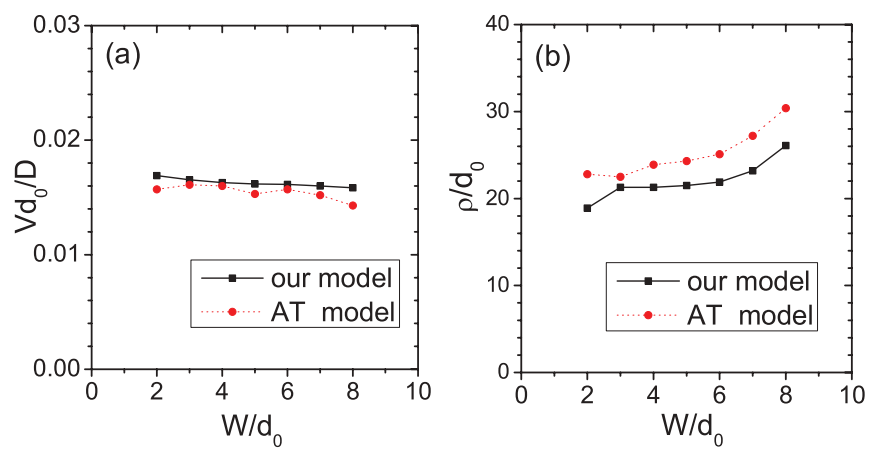

FIG. 3. (Color online) The dimensionless (a) tip velocity $V d_{0} / D$ and (b) tip radius $\rho / d_{0}$ of the steady growth state as a function of the dimensionless interface width, $W / d_{0}$.

much from the value obtained by the AT model for the smallest $W$. It is shown that with the increase of $\chi$ the tip velocity decreases and the tip radius increases, both of which are more sensitive to $\chi$ for larger $\mathrm{W}$. This agrees with our expectation because higher-order contributions become more significant for larger W. We plot in Fig. 3 the tip velocity and radius as a function of $W / d_{0}$. Because the simulation time scales $\sim\left(d_{0} / W\right)^{5}$, it is of practical importance to achieve reasonably good results at relatively large W. For $W=2 d_{0}$ we use the fixed TI value $\chi=0.4258$ for reference, while for $w=3 \sim 8 d_{0}$ we choose an appropriate value of $\chi$ to yield the tip velocity in good agreement with that at $W=2 d_{0}$. Obviously, at $W=2 d_{0}$ (with no adjustable parameter) our model predicts a larger tip velocity and a smaller tip radius than the AT model. This is the consequence of the mesoscopic solute drag effect included in our model and neglected in the AT model. The AT model also shows good convergent behavior for the tip velocity, but the predicted tip radius blows up more quickly than our model for large $\mathrm{W}$. With $\tau_{0}$ exactly specified by the TI result [Eq. (16)], its good performance on predicting the tip velocity at the intermediate range of $W / d_{0}$ is probably due to cancellation of errors by simultaneously using an underestimated phase relaxation time and neglecting the mesoscopic solute drag effect. We note that if if we increase $\mathrm{W}$ to $10 d_{0}$, it becomes impossible to find a good $\chi$ that both avoids solute trapping and gives reasonably large tip velocity. This indicates it is too far away from the TI regime, and the form of kinetic current (linearly proportional to $W$ ) is no longer adequate.

It is also important to examine the solute profile in the solid. By definition we have $\left[c_{s}(x) / c_{l}^{0}-k\right] / k=(1-k) u(x)$, so by looking at the profile of $u$ it is rather straightforward to find how well the solute distribution obeys the equilibrium partition rule. In fact, in all of the cases presented here, we find the solute concentration in the solid consistently agrees within a couple percentage points with the Gibbs-Thomson relation $c_{s}(x) / c_{l}^{0}=k\left[1-(1-k) d_{0} / \rho\right]$. Therefore our model is efficient to remove the artificial solute trapping and at the same time yields accurate results on tip velocity and radius, even for $W=7 d_{0}$.

\section{CONCLUSIONS}

In conclusion, we have developed a thermodynamically consistent variational phase-field model capable of quantitatively simulating microstructural pattern formation in alloys. The mesoscopic solute drag effect, not captured by the phenomenological AT model, is naturally recovered within the framework of LIT. Simulations on 2D isothermal dendrite growth show the capability of our model to quantitatively describe alloy solidification. Importantly, our model can be extended to describe pattern formation in alloys with nonzero solid diffusivity and nontrivial thermosolutal coupling [18], multiphase and multicomponent systems, and a wide range of other interfacial dynamic phenomena.

Note added: We are aware of the very recent work by Brener and Boussinot [19], in which a similar phase-field model is proposed.

\section{ACKNOWLEDGMENT}

The authors are grateful to A.-C. Shi and Z.-G. Wang for discussions and M. Plapp for a critical reading of the manuscript. The research grant to Yongli Mi by the China 985 research grant is greatly appreciated for the support of this work.
[1] J. B. Collins and H. Levine, Phys. Rev. B 31, 6119 (1985); G. Caginalp and P. Fife, ibid. 33, 7792 (1986).

[2] J. S. Langer, in Directions in Condensed Matter, edited by G. Grinstein and G. Mazenko (World Scientific, Singapore, 1986), p. 164.

[3] L.-Q. Chen, Annu. Rev. Mater. Res. 32, 113 (2002); W. J. Boettinger, J. A. Warren, C. Beckermann, and A. Karma, ibid. 32, 163 (2002)

[4] A. A. Wheeler, W. J. Boettinger, and G. B. McFadden, Phys. Rev. A 45, 7424 (1992); Phys. Rev. E 47, 1893 (1993); G. Caginalp and W. Xie, ibid. 48, 1897 (1993); R. Kobayashi, Physica D 63, 410 (1993); J. A. Warren and W. J. Boettinger, Acta Metall. Mater. 43, 689 (1995); J. Tiaden, B. Nestler, H.-J. Diepers, and I. Steinbach, Physica D 115, 73 (1998).
[5] A. Karma and W.-J. Rappel, Phys. Rev. E 53, R3017 (1996); 57, 4323 (1998).

[6] R. F. Almgren, SIAM J. Appl. Math. 59, 2086 (1999).

[7] A. Karma, Phys. Rev. Lett. 87, 115701 (2001).

[8] M. Ohno and K. Matsuura, Phys. Rev. E 79, 031603 (2009).

[9] S. G. Kim, Acta Mater. 55, 4391 (2007).

[10] W. Kurz and D. J. Fisher, Fundamentals of Solidification (Trans Tech, Aedermannsdorf, Switzerland, 1992).

[11] S. G. Kim, W. T. Kim, and T. Suzuki, Phys. Rev. E 60, 7186 (1999).

[12] E. A. Brener and D. E. Temkin, Phys. Rev. E 85, 031601 (2012).

[13] S.-L. Wang, R. F. Sekerka, A. A. Wheeler, B. T. Murray, S. R. Coriell, R. J. Braun, and G. B. McFadden, Physica D 69, 189 (1993). 
[14] B. Castaing and P. Nozieres, J. Phys. (Paris) 41, 701 (1980).

[15] S. Balibar, D. O. Edwards, and W. F. Saam, J. Low Temp. Phys. 82, 119 (1991).

[16] B. Echebarria, R. Folch, A. Karma, and M. Plapp, Phys. Rev. E 70, 061604 (2004).

[17] The introducing of such an adjustable parameter seems to be an unpleasant feature, as compared to previous models with the phase relaxation time fully determined by TI analysis.
However, with the parameters used in our simulation $(u=$ $-0.55)$, together with the presence of the mesoscopic solute drag term, we do not expect the second-order asymptotic result is sufficiently accurate at $W / d_{0}>1$ (M. Plapp, private communication).

[18] J. C. Ramirez, C. Beckermann, A. Karma, and H.-J. Diepers, Phys. Rev. E 69, 051607 (2004).

[19] E. A. Brener and G. Boussinot, Phys. Rev. E 86, 060601(R) (2012). 